\title{
Reconstruction of Historic Landscapes
}

\author{
Paul Richens \\ University of Bath \\ BA2 7AY UK \\ pnr20@bath.ac.uk
}

Marion Harney
University of Bath
BA2 7AY UK
abpmh@bath.ac.uk

handle more than $8 \mathrm{~km}$. The solution was to use an image-warping technique to apply hyperbolic compression to a digital terrain model, and associated aerial photography. This kept the central $2 \mathrm{~km}$ radius true to scale, but applied a kind of perspective shrinking of the remainder to fit within a bowl of $4 \mathrm{~km}$ radius. The view from the centre of the projection looks exactly the same.

The landscape rendering required a large number of trees, of recognisable English and exotic species. We developed a parametric generator in Processing to manufacture several versions of each species, each with several levels of detail obtained by using Catmull-Clark subdivision. Coupled with stereo photography of bark and foliage, we were able to place some tens of thousands of realistic trees in the landscape.

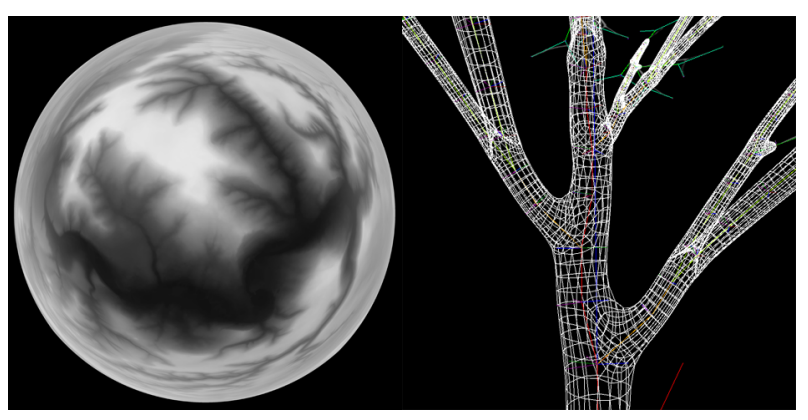

Figure 2: Terrain model after hyperbolic compression

Figure 3: Tree modelled as a subdivision surface

\section{RESULTS}

The completed game can be presented in several different formats. The simplest is to play it on a desktop computer, with keyboard and mouse. A darkened room and a pair of earphones improve the quality.

We have shown it quite often in a lecture-room setting, using a large-screen projection. This can be quite impressive, but viewers do not have the 
chance to interact and explore on their own. Most of them will not be seated in the ideal viewing position, and the presenter has to drive with caution, otherwise the audience may suffer mild giddiness.

Our preferred presentation is to project from overhead onto a large screen, reaching from floor level to 3 metres above, in a dark space. A lectern is placed at the exact centre of perspective, with a tethered game-controller and some basic instructions. Audio is channelled through a $5+1$ surround system. Placing the player at the exact centre of the visual and audio simulation gives a substantial boost to the feeling of immersion.

Displayed in this way, we achieve our primary aim, which was to generate interpretive exhibition material for public consumption, to convey in a fully rounded and immersive form what a lost landscape would have been like to enter and walk around, and to relate this visual impression to the intentions and taste of the creator, and also to the surviving scene of everyday experience.

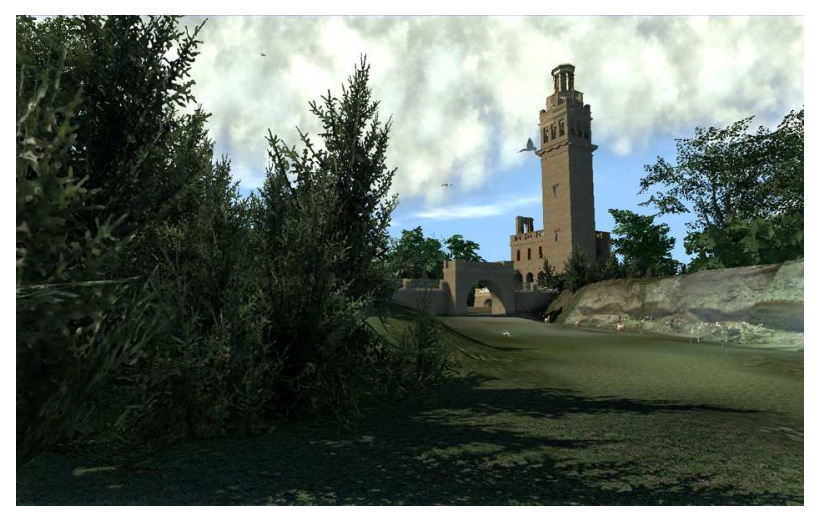

Figure 1: Beckford's Tower in a reconstructed landscape
2. acknowledgements

The project was funded by the Beckford Tower Trust with the help of a grant from the Heritage Lottery Fund. Architectural modelling by James Bailey, Joe Mummery, and Stephan WassermanFry, programming Paul Shepherd and Simon Hartley. CryEngine 3 was provided under an academic license from Crytek GmbH Frankfurt.

\section{REFERENCES}

Catmull, E., and Clark, J.(1978) Recursively generated bspline surfaces on arbitrary topological meshes. Computer Aided Design 10, 350-355.

Fothergill, B. (1979) Beckford of Fonthill. Faber and Faber, London.

Lansdown, H.V. (1893) Recollections of the late William Beckford

Maddox, W. (1844). Views of Lansdown Tower, Bath, the favourite edifice of the late William Beckford. Bath, E.English.

Sousa, T.(2011) Crysis 2 \& CryENGINE 3, Key Rendering Features. Crytek.

http://crytek.com/assets/Crysis-2-Key-RenderingFeatures.pdf (2 April 2011) 